\title{
HARMONIC INTERPOLATING WAVELETS IN NEUMANN BOUNDARY VALUE PROBLEM IN A CIRCLE
}

\author{
Dmitry A. Yamkovoi \\ Krasovskii Institute of Mathematics and Mechanics, \\ Ural Branch of the Russian Academy of Sciences, \\ 16 S. Kovalevskaya str., Ekaterinburg, Russia, 620990 \\ dmitriiyamkovoi@bk.ru
}

\begin{abstract}
The Neumann boundary value problem (BVP) in a unit circle is discussed. For the solution of the Neumann BVP, we built a method employing series representation of given $2 \pi$-periodic continuous boundary function by interpolating wavelets consisting of trigonometric polynomials. It is convenient to use the method due to the fact that such series is easy to extend to harmonic polynomials inside a circle. Moreover, coefficients of the series have an easy-to-calculate form. The representation by the interpolating wavelets is constructed by using an interpolation projection to subspaces of a multiresolution analysis with basis $2 \pi$-periodic scaling functions (more exactly, their binary rational compressions and shifts). That functions were developed by Subbotin and Chernykh on the basis of Meyer-type wavelets. We will use three kinds of such functions, where two out of the three generates systems, which are orthogonal and simultaneous interpolating on uniform grids of the corresponding scale and the last one generates only interpolating on the same uniform grids system. As a result, using the interpolation property of wavelets mentioned above, we obtain the exact representation of the solution for the Neumann BVP by series of that wavelets and numerical bound of the approximation of solution by partial sum of such series.
\end{abstract}

Keywords: Wavelets, Interpolating wavelets, Harmonic functions, Neumann boundary value problem.

\section{Introduction}

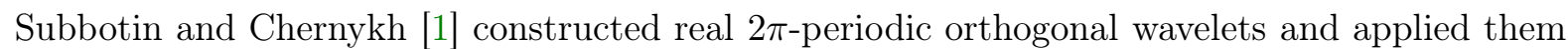
to represent and analyze solutions of Dirichlet, Neumann, and Poisson boundary value problems for harmonic and biharmonic functions. In [2] the Dirichlet BVP in a unit circle was solved by means of interpolating-orthogonal periodic wavelets from [3]. In the present paper, we propose to use the same wavelets for solving the Neumann BVP in a unit circle. Moreover, our main interest is the exact representation of the solution for the Neumann BVP by series of wavelet bases and behavior of partial sums of such series. For the sake of convenience, we give the reader an adequate background for further study and partially repeat sections with interpolating and interpolating-orthogonal $2 \pi$ periodic wavelet construction from $[1,3]$.

\section{Preliminaries}

Consideration of autocorrelation functions for orthonormal scaling functions instead of orthonormal scaling functions is commonly used construction technique for interpolating wavelets in $\mathbb{R}$. It is equivalent to replacement of scaling $\varphi(x)$ function by function, which Fourier transform coincides with $|\widehat{\varphi}(\omega)|^{2}$.

Let $\varepsilon$ be a fixed number from $(0,1 / 3]$ and let $\widehat{\varphi}_{\varepsilon}(\omega)$ be a Fourier transform of Meyer-type (see $[4,5])$ function:

$$
\left\{\begin{array}{cl}
\widehat{\varphi}_{\varepsilon}(\omega)=0, & |\omega|>(1+\varepsilon) / 2 \\
\widehat{\varphi}_{\varepsilon}(\omega)=1, & |\omega| \leq(1-\varepsilon) / 2 \\
\widehat{\varphi}_{\varepsilon}^{2}(\omega)+\widehat{\varphi}_{\varepsilon}^{2}(\omega-1)=1, & (1-\varepsilon) / 2<\omega<(1+\varepsilon) / 2 .
\end{array}\right.
$$


We also require that the function $\widehat{\varphi}_{\varepsilon}^{2}(\omega)$ is even and smooth on $\mathbb{R}$ with the symmetry center of its graph on the interval $((1-\varepsilon) / 2,(1+\varepsilon) / 2)$ at the point $\omega=1 / 2$. Define functions $\varphi_{s}(x)(s=1,2)$ as in [1] and function $\varphi_{3}(x)$ as in [2] such that:

$$
\begin{gathered}
\widehat{\varphi}_{1}(\omega)=\frac{1}{2}\left\{\begin{array}{l}
\left(1+\widehat{\varphi}_{\varepsilon}(\omega)-\widehat{\varphi}_{\varepsilon}(\omega-1)-\widehat{\varphi}_{\varepsilon}(\omega+1)\right)+i(\operatorname{sign} \omega) \sqrt{2 \beta(\omega)}, \\
|\omega|<(1+\varepsilon) / 2, \\
0, \\
|\omega| \geq(1+\varepsilon) / 2,
\end{array}\right. \\
\widehat{\varphi}_{2}(\omega)=\widehat{\varphi}_{\varepsilon}^{2}(\omega)+i(\operatorname{sign} \omega) \beta(\omega), \quad \beta(\omega)=\widehat{\varphi}_{\varepsilon}(\omega)\left(\widehat{\varphi}_{\varepsilon}(\omega-1)+\widehat{\varphi}_{\varepsilon}(\omega+1)\right), \\
\widehat{\varphi}_{3}(\omega)=\widehat{\varphi}_{\varepsilon}^{2}(\omega) .
\end{gathered}
$$

Here $\beta(\omega)$ is a smooth even function on $\mathbb{R}$ vanishing together with its derivative at the points $\omega=(-1 \pm \varepsilon) / 2$ and $\omega=(1 \pm \varepsilon) / 2$, with the support $\{((-1-\varepsilon) / 2,(-1+\varepsilon) / 2) \cup((1-\varepsilon) / 2,(1+\varepsilon) / 2)\}$ and even on intervals $\pm((1-\varepsilon) / 2,(1+\varepsilon) / 2)$ with respect to their centers $\omega= \pm 1 / 2$. Functions $\varphi_{s}(x)(s=1,2,3)$ generates interpolating in $C(\mathbb{R})$ systems $\left\{\varphi_{s}\left(2^{j} x-k\right): k \in \mathbb{Z}\right\}(j \in \mathbb{Z})$ on the grids $\left\{l / 2^{j}: l \in \mathbb{Z}\right\}(j \in \mathbb{Z})$. For $s=1,2$ these systems are also orthogonal in $L^{2}(\mathbb{R})$. Unless otherwise stipulated, throughout the paper $s=1,2,3$.

The 1-periodization process of the function $\varphi_{s}\left(2^{j} x\right)$

$$
\operatorname{Pe}_{1} \varphi_{s}\left(2^{j} x\right)=\sum_{\mu \in \mathbb{Z}} \varphi_{s}\left(2^{j}(x+\mu)\right)=: \Phi_{s}^{j, 0}(2 \pi x), \quad j \in \mathbb{Z}
$$

converges uniformly on the interval $[-1 / 2,1 / 2]$ (see [1]). Calculating the coefficients $a_{\nu}$ in the expansion of the function $\Phi_{s}^{j, 0}(2 \pi x)$ by the trigonometric system $\left\{e^{2 \pi i \nu x}: \nu \in \mathbb{Z}\right\}$, we get

$$
\Phi_{s}^{j, 0}(2 \pi x)=\sum_{\nu \in \mathbb{Z}} a_{\nu} e^{2 \pi i \nu x}, \quad j \in \mathbb{Z} .
$$

Using (1.1), we find all coefficients $a_{\nu}(\nu \in \mathbb{Z})$

$$
\begin{gathered}
a_{\nu}=\int_{0}^{1} \sum_{\mu \in \mathbb{Z}} \varphi_{s}\left(2^{j}(x+\mu)\right) e^{-2 \pi i \nu x} d x=\sum_{\mu \in \mathbb{Z}} \int_{0}^{1} \varphi_{s}\left(2^{j}(x+\mu)\right) e^{-2 \pi i \nu x} d x= \\
=[\text { substitution: } x+\mu=t] \sum_{\mu \in \mathbb{Z}} \int_{\mu}^{\mu+1} \varphi_{s}\left(2^{j} t\right) e^{-2 \pi i \nu(t-\mu)} d t= \\
=\int_{\mathbb{R}} \varphi_{s}\left(2^{j} t\right) e^{-2 \pi i \nu t} d t=\widehat{\varphi_{s}\left(2^{j} t\right)}=2^{-j} \widehat{\varphi}_{s}\left(\frac{\nu}{2^{j}}\right) .
\end{gathered}
$$

Substituting the coefficients $a_{\nu}(\nu \in \mathbb{Z})$ in (1.2), we obtain:

$$
\Phi_{s}^{j, 0}(2 \pi x)=2^{-j} \sum_{\nu \in \Delta_{\varepsilon}^{j} \cap \mathbb{Z}} \widehat{\varphi}_{s}\left(\frac{\nu}{2^{j}}\right) e^{2 \pi i \nu x}, \quad j \in \mathbb{Z},
$$

where $\Delta_{\varepsilon}^{j}=2^{j}((-1-\varepsilon) / 2,(1+\varepsilon) / 2)$. Replacing the variable $x$ by $x /(2 \pi)$, we obtain $2 \pi$-periodic wavelet systems

$$
\left\{\Phi_{s}^{j, k}(x):=\Phi_{s}^{j, 0}\left(x-\frac{2 \pi k}{2^{j}}\right)=2^{-j} \sum_{\nu \in \Delta_{\varepsilon}^{j} \cap \mathbb{Z}} \widehat{\varphi}_{s}\left(\frac{\nu}{2^{j}}\right) e^{i \nu\left(x-2 \pi k / 2^{j}\right)}: k \in \mathbb{Z}\right\}, \quad j \in \mathbb{Z},
$$


which are interpolating on the grids $\left\{x_{j}^{l}:=2 \pi l / 2^{j}: l=\overline{0,2^{j}-1}\right\}$ for $s=1,2,3$ and orthogonal in $L^{2}(\mathbb{R})$ for $s=1,2$.

It is easy to see, that for $n \in \mathbb{Z}$

$$
\Phi_{s}^{j, k+2^{j} n}(x)=2^{-j} \sum_{\nu \in \Delta_{\varepsilon}^{j} \cap \mathbb{Z}} \widehat{\varphi}_{s}\left(\frac{\nu}{2^{j}}\right) e^{i \nu\left(x-2 \pi k / 2^{j}-2 \pi n\right)}=\Phi_{s}^{j, k}(x) .
$$

So the sequence of spaces (1.3) has only $2^{j}$ distinct linearly independent terms. Hence, we can assume in the following discussion that $k=\overline{0,2^{j}-1}$.

Define system of spaces $\left\{V_{s}^{j}:=\operatorname{span}\left\{\Phi_{s}^{j, k}(x): k=\overline{0,2^{j}-1}\right\}: j \in \mathbb{Z}\right\}$. As follows from $\Delta_{\varepsilon}^{j} \cap \mathbb{Z}=\{0\}$ for $j \leq 0$ and $\widehat{\varphi}_{s}(0)=1$, we see that

$$
\Phi_{s}^{0,0}(x)=\sum_{\nu \in \Delta_{\varepsilon}^{0} \cap \mathbb{Z}} \widehat{\varphi}_{s}(\nu) e^{i \nu x}=1
$$

and

$$
\Phi_{s}^{j, 0}(x)=2^{-j} \sum_{\nu \in \Delta_{\varepsilon}^{j} \cap \mathbb{Z}} \widehat{\varphi}_{s}\left(\frac{\nu}{2^{j}}\right) e^{i \nu x}=2^{-j}, \quad j<0,
$$

i.e., for all integers such that $j \leq 0$ and for all $k \in \mathbb{Z}$ relation $\Phi_{s}^{j, k}(x)=\Phi_{s}^{j, 0}(x)=$ const holds and thus we can consider the system of spaces $\left\{V_{s}^{j}\right\}$ only for $j \in \mathbb{N} \cup\{0\}$. Further, for $j \in \mathbb{N} \cup\{0\}$ define spaces $W_{s}^{j}$ as direct complement of $V_{s}^{j}$ to $V_{s+1}^{j}$ with the interpolation system $\left\{\Psi_{s}^{j, k}(x): k=\overline{0,2^{j}-1}\right\}$ on the grid $\left\{x_{j+1}^{2 l+1}: l=\overline{0,2^{j}-1}\right\}$, which is interpolating basis of $2 \pi$-periodic continuous functions.

Show that the $\Psi_{s}^{j, k}(x)=\Phi_{s}^{j+1,2 k+1}(x)$ holds for all $j \in \mathbb{N} \cup\{0\}$ and for all $k=\overline{0,2^{j}-1}$. Since $V_{s}^{j} \subset V_{s}^{j+1}(j \in \mathbb{N} \cup\{0\})$, we see that

$$
\Phi_{s}^{j, k}(x)=\sum_{n=0}^{2^{j+1}-1} b_{n} \Phi_{s}^{j+1, n}(x), \quad j \in \mathbb{N} \cup\{0\}, \quad k=\overline{0,2^{j}-1} .
$$

Using interpolating condition of basis $\left\{\Phi_{s}^{j+1, k}(x): k=\overline{0,2^{j+1}-1}\right\}$ on the grid $\left\{x_{j+1}^{l}: l=\overline{0,2^{j+1}-1}\right\}$ and assuming $x:=2 \pi l / 2^{j+1}$ in (1.4), we find the coefficients $b_{n}\left(n=\overline{0,2^{j+1}-1}\right)$ :

$$
\Phi_{s}^{j, k}\left(\frac{2 \pi l}{2^{j+1}}\right)=\sum_{n=0}^{2^{j+1}-1} b_{n} \Phi_{s}^{j+1, n}\left(\frac{2 \pi l}{2^{j+1}}\right)=\sum_{n=0}^{2^{j+1}-1} b_{n} \delta_{n, l}, \quad l=\overline{0,2^{j+1}-1}
$$

so

$$
b_{n}=\Phi_{s}^{j, k}\left(\frac{2 \pi n}{2^{j+1}}\right), \quad n=\overline{0,2^{j+1}-1}
$$

In view of $b_{n}$ obtained, the sum on the right side of the expression (1.4) may be written as two sums over even and odd indices

$$
\begin{gathered}
\Phi_{s}^{j, k}(x)=\sum_{n=0}^{2^{j+1}-1} \Phi_{s}^{j, k}\left(\frac{2 \pi n}{2^{j+1}}\right) \Phi_{s}^{j+1, n}(x)=\sum_{n=0}^{2^{j}-1} \Phi_{s}^{j, k}\left(\frac{2 \pi n}{2^{j}}\right) \Phi_{s}^{j+1,2 n}(x)+ \\
+\sum_{n=0}^{2^{j}-1} \Phi_{s}^{j, k}\left(\frac{2 \pi(2 n+1)}{2^{j+1}}\right) \Phi_{s}^{j+1,2 n+1}(x)=\underbrace{\Phi_{s}^{j, k}\left(\frac{2 \pi k}{2^{j}}\right)}_{=1} \Phi_{s}^{j+1,2 k}(x)+ \\
+\sum_{n=0}^{2^{j}-1} \Phi_{s}^{j, k}\left(\frac{2 \pi(2 n+1)}{2^{j+1}}\right) \Phi_{s}^{j+1,2 n+1}(x) .
\end{gathered}
$$


As a result, we have

$$
\Phi_{s}^{j, k}(x)=\Phi_{s}^{j+1,2 k}(x)+\sum_{n=0}^{2^{j}-1} \Phi_{s}^{j, k}\left(\frac{2 \pi(2 n+1)}{2^{j+1}}\right) \Phi_{s}^{j+1,2 n+1}(x), \quad j \in \mathbb{N} \cup\{0\}, \quad k=\overline{0,2^{j}-1}
$$

i.e.,

$$
\underbrace{\Phi_{s}^{j+1,2 k}(x)}_{\in V_{s}^{j+1}}=\underbrace{\Phi_{s}^{j, k}(x)}_{\in V_{s}^{j}}-\sum_{n=0}^{2^{j}-1} \Phi_{s}^{j, k}\left(\frac{2 \pi(2 n+1)}{2^{j+1}}\right) \Phi_{s}^{j+1,2 n+1}(x), \quad j \in \mathbb{N} \cup\{0\}, \quad k=\overline{0,2^{j}-1},
$$

and it implies that

$$
\begin{gathered}
V_{s}^{j+1}=\left\{\sum_{k=0}^{2^{j+1}-1} c_{j+1, k} \Phi_{s}^{j+1, k}(x): c_{j+1, k} \in \mathbb{R}\right\}= \\
=\left\{\sum_{k=0}^{2^{j}-1} c_{j+1,2 k} \Phi_{s}^{j+1,2 k}(x)+\sum_{k=0}^{2^{j}-1} c_{j+1,2 k+1} \Phi_{s}^{j+1,2 k+1}(x)\right\}=\left\{\sum_{k=0}^{2^{j}-1} c_{j+1,2 k} \Phi_{s}^{j, k}(x)-\right. \\
\left.-\sum_{k=0}^{2^{j}-1} c_{j+1,2 k} \sum_{n=0}^{2^{j}-1} \Phi_{s}^{j, k}\left(\frac{2 \pi(2 n+1)}{2^{j+1}}\right) \Phi_{s}^{j+1,2 n+1}(x)+\sum_{k=0}^{2^{j}-1} c_{j+1,2 k+1} \Phi_{s}^{j+1,2 k+1}(x)\right\}= \\
=\left\{\sum_{k=0}^{2^{j}-1} c_{j+1,2 k} \Phi_{s}^{j, k}(x)-\sum_{n=0}^{2^{j}-1} \Phi_{s}^{j+1,2 n+1}(x) \sum_{k=0}^{2^{j}-1} c_{j+1,2 k} \Phi_{s}^{j, k}\left(\frac{2 \pi(2 n+1)}{2^{j+1}}\right)+\right. \\
\left.+\sum_{n=0}^{2^{j}-1} c_{j+1,2 n+1} \Phi_{s}^{j+1,2 n+1}(x)\right\}=\left\{\sum_{k=0}^{2^{j}-1} c_{j+1,2 k} \Phi_{s}^{j, k}(x)+\sum_{n=0}^{2^{j}-1} d_{j, n} \Phi_{s}^{j+1,2 n+1}(x):\right. \\
\left.d_{j, n}=-\sum_{k=0}^{2^{j}-1} c_{j+1,2 k} \Phi_{s}^{j, k}\left(\frac{2 \pi(2 n+1)}{2^{j+1}}\right)+c_{j+1,2 n+1}\right\}=V_{s}^{j} \oplus W_{s}^{j} .
\end{gathered}
$$

In view of definitions of spaces $V_{s}^{j}$ and $W_{s}^{j}$, for all $j \in \mathbb{N} \cup\{0\}$ and for all $k=\overline{0,2^{j}-1}$ relation

$$
\Psi_{s}^{j, k}(x)=\Phi_{s}^{j+1,2 k+1}(x)
$$

holds.

Denote the interpolation projection of a function $f \in C_{2 \pi}$ (the space of continuous $2 \pi$-periodic functions) onto the $V_{s}^{j}$ by

$$
S_{s, 2^{j}}(x ; f)=\sum_{k=0}^{2^{j}-1} f\left(\frac{2 \pi k}{2^{j}}\right) \Phi_{s}^{j, k}(x), \quad j \in \mathbb{N} \cup\{0\} .
$$

Since $\overline{\bigcup_{j=0}^{\infty} V_{s}^{j}}=C_{2 \pi}$, for $f \in C_{2 \pi}$ we have

$$
\begin{gathered}
S_{s, 2^{j}}(x ; f) \underset{\mathbb{R}}{\rightrightarrows} f(x), \\
f(x)=f(0)+\sum_{j=0}^{+\infty} \sum_{k=0}^{2^{j}-1} c_{j, k} \Psi_{s}^{j, k}(x)=f(0)+\sum_{j=0}^{+\infty} \sum_{k=0}^{2^{j}-1} c_{j, k} \Phi_{s}^{j+1,2 k+1}(x) .
\end{gathered}
$$


Find all coefficients $c_{j, k},\left(j \in \mathbb{N} \cup\{0\}, k=\overline{0,2^{j}-1}\right)$ from (1.7). Because of $S_{s, 2^{j}}(x ; f) \in V_{s}^{j}$, $S_{s, 2^{j+1}}(x ; f) \in V_{s}^{j+1}$ and definition of spaces $W_{s}^{j}$ we have $S_{s, 2^{j+1}}(x ; f)-S_{s, 2^{j}}(x ; f) \in W_{s}^{j}$, i.e.,

$$
\left.\left(S_{s, 2^{j+1}}(x ; f)-S_{s, 2^{j}}(x ; f)\right)\right|_{x=x_{j+1}^{2 l+1}}=\sum_{k=0}^{2^{j}-1} c_{j, k} \Phi_{s}^{j+1,2 k+1}\left(x_{j+1}^{2 l+1}\right)=\sum_{k=0}^{2^{j}-1} c_{j, k} \delta_{k, l}=c_{j, l},
$$

where $j \in \mathbb{N} \cup\{0\}$ and $l=\overline{0,2^{j}-1}$. Using definition (1.5), we rewrite $S_{s, 2^{j+1}}(x ; f)$ and take $x:=x_{j+1}^{2 l+1}$

$$
\left.S_{s, 2^{j+1}}(x ; f)\right|_{x=x_{j+1}^{2 l+1}}=\sum_{k=0}^{2^{j+1}-1} f\left(\frac{2 \pi k}{2^{j+1}}\right) \Phi_{s}^{j+1, k}\left(x_{j+1}^{2 l+1}\right)=\sum_{k=0}^{2^{j+1}-1} f\left(\frac{2 \pi k}{2^{j+1}}\right) \delta_{k, 2 l+1}=f\left(x_{j+1}^{2 l+1}\right) .
$$

Consequently,

$$
c_{j, l}=S_{s, 2^{j+1}}\left(x_{j+1}^{2 l+1} ; f\right)-S_{s, 2^{j}}\left(x_{j+1}^{2 l+1} ; f\right)=f\left(x_{j+1}^{2 l+1}\right)-S_{s, 2^{j}}\left(x_{j+1}^{2 l+1} ; f\right), \quad j \in \mathbb{N} \cup\{0\}, \quad l=\overline{0,2^{j}-1} .
$$

With (1.3), (1.7) and preceding expression the following relation holds for a function $f \in C_{2 \pi}$

$$
\begin{gathered}
f(x)=f(0)+\sum_{j=0}^{+\infty} \sum_{k=0}^{2^{j}-1} c_{j, k} \Psi_{s}^{j, k}(x)=f(0)+\sum_{j=0}^{+\infty} \sum_{k=0}^{2^{j}-1}\left(f\left(x_{j+1}^{2 k+1}\right)-S_{s, 2^{j}}\left(x_{j+1}^{2 k+1} ; f\right)\right) \times \\
\times \Phi_{s}^{j+1,2 k+1}(x)=f(0)+\sum_{j=0}^{+\infty} \sum_{k=0}^{2^{j}-1}\left(f\left(x_{j+1}^{2 k+1}\right)-S_{s, 2^{j}}\left(x_{j+1}^{2 k+1} ; f\right)\right) 2^{-(j+1)} \times \\
\times \sum_{\nu \in \Delta_{\varepsilon}^{j+1} \cap \mathbb{Z}} \widehat{\varphi}_{s}\left(\frac{\nu}{2^{j+1}}\right) e^{i \nu\left(x-2 \pi(2 k+1) / 2^{j+1}\right)} .
\end{gathered}
$$

The definition of $W_{s}^{j}$ imply $V_{s}^{j}=V_{s}^{0} \oplus\left(\oplus_{l=0}^{j-1} W_{s}^{l}\right)$. Then $S_{s, 2^{j}}(x ; f)$ is the partial sum of order $2^{j}$ for (1.7) and from (1.6) series (1.7) converges uniformly. Thus for $J \in \mathbb{Z}$

$$
S_{s, 2^{J}}(x ; f)=f(0)+\sum_{j=0}^{J-1} \sum_{k=0}^{2^{j}-1}\left(f\left(x_{j+1}^{2 k+1}\right)-S_{s, 2^{j}}\left(x_{j+1}^{2 k+1} ; f\right)\right) \Phi_{s}^{j+1,2 k+1}(x)
$$

and as $J \rightarrow \infty$

$$
S_{s, 2^{J}}(x ; f) \underset{\mathbb{R}}{\rightrightarrows} f(x)
$$

\section{Application to the solution of the Neumann BVP in a circle}

Setting of the Neumann BVP in the unit circle $K_{1}$ (see, for example, [6]):

$$
\left\{\begin{array}{l}
\Delta U(r, x)=\frac{\partial^{2} U}{\partial r^{2}}+\frac{1}{r} \frac{\partial U}{\partial r}+\frac{1}{r^{2}} \frac{\partial^{2} U}{\partial x^{2}}=0, \quad U \in C^{(1)}\left(\overline{K_{1}}\right) \cap C^{(2)}\left(K_{1}\right), \\
\frac{\partial U}{\partial r}(1, x)=g_{1}(x) \in C_{2 \pi},
\end{array}\right.
$$

where $r e^{i x}(0 \leq r<1,0 \leq x<2 \pi)$ are points of the unit circle $K_{1}$ centered at the origin of the polar coordinate system. It has been well known that necessary condition of solvability of the Neumann problem is

$$
\int_{0}^{2 \pi} g_{1}(x) d x=0
$$


and the problem have a unique solution up to an additive constant.

Define harmonic in the unit circle polynomials $\Phi_{s}^{j, k}(r, x)$ :

$$
\Phi_{s}^{j, k}(r, x):=2^{-j} \sum_{\nu \in \Delta_{\varepsilon}^{j} \cap \mathbb{Z}} \widehat{\varphi}_{s}\left(\frac{\nu}{2^{j}}\right) r^{|\nu|} e^{i \nu\left(x-2 \pi k / 2^{j}\right)}, \quad j \in \mathbb{N} \cup\{0\}, \quad k=\overline{0,2^{j}-1}
$$

and consider series

$$
U(1,0)+\sum_{j=0}^{+\infty} \sum_{k=0}^{2^{j}-1}\left(U(1, \cdot)-S_{s, 2^{j}}(1, \cdot ; U(1, \cdot))\right)\left(x_{j+1}^{2 k+1}\right) \Phi_{s}^{j+1,2 k+1}(1, x) .
$$

Since $U(r, x)$ is a harmonic in the unit circle function with continuous boundary value $U(1, x)$, it follows that the above series converges uniformly on the boundary of $K_{1}$ by taking into account (1.8) and (1.9) (where for $f(x)$ we take $U(1, x)$ ). Because of maximum principle for harmonic functions, we obtain the following representation for $U(r, x)$ in form of uniformly convergent in $\bar{K}_{1}$ series

$$
\begin{aligned}
U(r, x)=U(1,0)+ & \sum_{j=0}^{+\infty} \sum_{k=0}^{2^{j}-1}\left(U(1, \cdot)-S_{s, 2^{j}}(1, \cdot ; U(1, \cdot))\right)\left(x_{j+1}^{2 k+1}\right) \Phi_{s}^{j+1,2 k+1}(r, x)= \\
=U(1,0)+ & \sum_{j=0}^{+\infty} \sum_{k=0}^{2^{j}-1}\left(U(1, \cdot)-S_{s, 2^{j}}(1, \cdot ; U(1, \cdot))\right)\left(x_{j+1}^{2 k+1}\right) 2^{-(j+1)} \times \\
& \times \sum_{\nu \in \Delta_{\varepsilon}^{j+1} \cap \mathbb{Z}} \widehat{\varphi}_{s}\left(\frac{\nu}{2^{j+1}}\right) r^{|\nu|} e^{i \nu\left(x-2 \pi(2 k+1) / 2^{j+1}\right)} .
\end{aligned}
$$

Using (1.8), we have the following representation for function $g_{1}(x) \in C_{2 \pi}$ in form of uniformly convergent in $\bar{K}_{1}$ series

$$
g_{1}(x)=g_{1}(0)+\sum_{j=0}^{+\infty} \sum_{k=0}^{2^{j}-1}\left(g_{1}(\cdot)-S_{s, 2^{j}}\left(\cdot ; g_{1}\right)\right)\left(x_{j+1}^{2 k+1}\right) 2^{-(j+1)} \sum_{\nu \in \Delta_{\varepsilon}^{j+1} \cap \mathbb{Z}} \widehat{\varphi}_{s}\left(\frac{\nu}{2^{j+1}}\right) e^{i \nu\left(x-2 \pi(2 k+1) / 2^{j+1}\right)} .
$$

We may extend terms of the series into the interior of the unit circle to harmonic polynomials $c_{j, k}\left(g_{1}\right) \Phi_{s}^{j, k}(r, x)$ and, consequently, we may extend the series into the interior of the unit circle to harmonic in $K_{1}$ and in continuous $\bar{K}_{1}$ function.

$$
\begin{aligned}
g_{1}(r, x):= & g_{1}(0)+\sum_{j=0}^{+\infty} \sum_{k=0}^{2^{j}-1}\left(g_{1}(\cdot)-S_{s, 2^{j}}\left(\cdot ; g_{1}\right)\right)\left(x_{j+1}^{2 k+1}\right) 2^{-(j+1)} \times \\
& \times \sum_{\nu \in \Delta_{\varepsilon}^{j+1} \cap \mathbb{Z}} \widehat{\varphi}_{s}\left(\frac{\nu}{2^{j+1}}\right) r|\nu| e^{i \nu\left(x-2 \pi(2 k+1) / 2^{j+1}\right)} .
\end{aligned}
$$

Because of series in (2.3) converges uniformly, we can perform a term-by-term differentiation with respect to $r$ and multiplication by $r$ and as result we get

$$
\begin{aligned}
r \frac{\partial U}{\partial r}(r, x) & =\sum_{j=0}^{+\infty} \sum_{k=0}^{2^{j}-1}\left(U(1, \cdot)-S_{s, 2^{j}}(1, \cdot ; U(1, \cdot))\right)\left(x_{j+1}^{2 k+1}\right) 2^{-(j+1)} \times \\
& \times \sum_{\nu \in \Delta_{\varepsilon}^{j+1} \cap \mathbb{Z}} \widehat{\varphi}_{s}\left(\frac{\nu}{2^{j+1}}\right)|\nu| r^{|\nu|} e^{i \nu\left(x-2 \pi(2 k+1) / 2^{j+1}\right)} .
\end{aligned}
$$


As is easy to see that this function is harmonic in $K_{1}$. In view of setting of the Neumann BVP, we have $\left.\frac{\partial U}{\partial r}(r, x)\right|_{r=1}=g_{1}(x)$, this implies that for $0 \leq r<1$ the equality $r \frac{\partial U}{\partial r}(r, x)=g_{1}(r, x)$ holds as equality of two harmonic functions which are equal at the boundary of $K_{1}$. Hence

$$
\begin{aligned}
r \frac{\partial U}{\partial r}(r, x) & =g_{1}(0)+\sum_{j=0}^{+\infty} \sum_{k=0}^{2^{j}-1}\left(g_{1}(\cdot)-S_{s, 2^{j}}\left(\cdot ; g_{1}\right)\right)\left(x_{j+1}^{2 k+1}\right) 2^{-(j+1)} \times \\
& \times \sum_{\nu \in \Delta_{\varepsilon}^{j+1} \cap \mathbb{Z}} \widehat{\varphi}_{s}\left(\frac{\nu}{2^{j+1}}\right) r^{|\nu|} e^{i \nu\left(x-2 \pi(2 k+1) / 2^{j+1}\right)} .
\end{aligned}
$$

In consequence of (2.2), we also have

$$
\int_{0}^{2 \pi} g_{1}(r, x) d x=0
$$

Indeed if we expand function $g_{1}(r, x)=r \frac{\partial U}{\partial r}(r, x)$ in a series by system $\left\{r^{|n|} e^{i n x}: n \in \mathbb{Z}\right\}$ (for instance, with the use of Poisson kernel), then we get for $0 \leq r<1$

$$
g_{1}(r, x)=\frac{1}{2 \pi} \int_{0}^{2 \pi} g_{1}(1, t) P_{r}(x-t) d t=\frac{1}{2 \pi} \int_{0}^{2 \pi} \sum_{n \in \mathbb{Z}} g_{1}(t) r^{|n|} e^{i n(x-t)} d t .
$$

Interchanging of integration and summation and using (2.2), we arrive at

$$
\sum_{n \in \mathbb{Z} \backslash\{0\}}\left(\frac{1}{2 \pi} \int_{0}^{2 \pi} g_{1}(1, t) e^{-i n t} d t\right) r^{|n|} e^{i n x},
$$

resulting in (2.5).

Thus, using (2.5) and taking into account $\widehat{\varphi}_{s}(0)=1$, we obtain

$$
g_{1}(0)+\sum_{j=0}^{+\infty} \sum_{k=0}^{2^{j}-1}\left(g_{1}(\cdot)-S_{s, 2^{j}}\left(\cdot ; g_{1}\right)\right)\left(x_{j+1}^{2 k+1}\right) 2^{-(j+1)}=0
$$

and numerical series on the left side of the equality converges. Consequently, the following equality holds

$$
g_{1}(r, x)=\sum_{j=0}^{+\infty} \sum_{k=0}^{2^{j}-1}\left(g_{1}(\cdot)-S_{s, 2^{j}}\left(\cdot ; g_{1}\right)\right)\left(x_{j+1}^{2 k+1}\right) 2^{-(j+1)} \sum_{\nu \in \Delta_{\varepsilon}^{j+1} \cap \mathbb{Z} \backslash\{0\}} \widehat{\varphi}_{s}\left(\frac{\nu}{2^{j+1}}\right) r^{|\nu|} e^{i \nu\left(x-2 \pi(2 k+1) / 2^{j+1}\right)} .
$$

Therefore, by setting

$$
\begin{gathered}
\Phi_{s}^{j+1,2 k+1,0}(r, x):=\Phi_{s}^{j+1,2 k+1}(r, x)-\frac{1}{2 \pi} \int_{0}^{2 \pi} \Phi_{s}^{j+1,2 k+1}(1, x) d x= \\
=2^{-(j+1)} \sum_{\nu \in \Delta_{\varepsilon}^{j+1} \cap \mathbb{Z} \backslash\{0\}} \widehat{\varphi}_{s}\left(\frac{\nu}{2^{j+1}}\right) r^{|\nu|} e^{i \nu\left(x-2 \pi(2 k+1) / 2^{j+1}\right)},
\end{gathered}
$$


we obtain

$$
\begin{gathered}
\frac{\partial U}{\partial r}(r, x)=\frac{g_{1}(r, x)}{r}=\sum_{j=0}^{+\infty} \sum_{k=0}^{2^{j}-1}\left(g_{1}(\cdot)-S_{s, 2^{j}}\left(\cdot ; g_{1}\right)\right)\left(x_{j+1}^{2 k+1}\right) \frac{\Phi_{s}^{j+1,2 k+1,0}(r, x)}{r}= \\
=\sum_{j=0}^{+\infty} \sum_{k=0}^{2^{j}-1}\left(g_{1}(\cdot)-S_{s, 2^{j}}\left(\cdot ; g_{1}\right)\right)\left(x_{j+1}^{2 k+1}\right) 2^{-(j+1)} \sum_{\nu \in \Delta_{\varepsilon}^{j+1} \cap \mathbb{Z} \backslash\{0\}} \widehat{\varphi}_{s}\left(\frac{\nu}{2^{j+1}}\right) r^{|\nu|-1} e^{i \nu\left(x-2 \pi(2 k+1) / 2^{j+1}\right)},
\end{gathered}
$$

where the series converges uniformly in $\bar{K}_{1}$. Setting

$$
\begin{gathered}
\Psi_{s}^{j, k,-1}(r, x):=\int_{0}^{r} \frac{\Phi_{s}^{j+1,2 k+1,0}\left(r^{\prime}, x\right)}{r^{\prime}} d r^{\prime}= \\
=2^{-(j+1)} \sum_{\nu \in \Delta_{\varepsilon}^{j+1} \cap \mathbb{Z} \backslash\{0\}} \widehat{\varphi}_{s}\left(\frac{\nu}{2^{j+1}}\right) \frac{r^{|\nu|}}{|\nu|} e^{i \nu\left(x-2 \pi(2 k+1) / 2^{j+1}\right)}, \quad j \in \mathbb{N} \cup\{0\}, \quad k=\overline{0,2^{j}-1},
\end{gathered}
$$

and calculating the $U(r, x)$ from the preceding equality, we formulate the following theorem.

Theorem 1. Under conditions of setting of the Neumann BVP (2.1) we obtain for $s=1,2,3$

$$
U(r, x)=U(1,0)+\sum_{j=0}^{+\infty} \sum_{k=0}^{2^{j}-1}\left(g_{1}(\cdot)-S_{s, 2^{j}}\left(\cdot ; g_{1}\right)\right)\left(x_{j+1}^{2 k+1}\right) \Psi_{s}^{j, k,-1}(r, x), \quad r e^{i x} \in \bar{K}_{1} .
$$

Series in (2.6) converges uniformly in $\bar{K}_{1}$ and $U(1,0)$ is a constant.

P r o o f follows from preceding equations.

Also we obtain the error for approximation of solution $U(r, x)$ of the problem (2.1) by partial sums of series $(2.6)$ denoted by $S_{s, 2^{J}}\left(r, x ; U, \Psi^{-1}\right)$. Denote by $E_{N_{\varepsilon, J}^{-}}(f)_{C_{2 \pi}}$ the best approximation of a function $f$ in $C_{2 \pi}$ by trigonometric polynomials of order $N_{\varepsilon, J}^{-}=\left\lfloor 2^{J-1}(1-\varepsilon)\right\rfloor$.

Theorem 2. Under conditions of setting of the Neumann BVP (2.1) for $s=1,2,3$ and $J \in Z_{+}:=\{j \in \mathbb{Z}: j \geq 0\}$ the function $S_{s, 2^{J}}(r, x ; U, \Psi)$ approximates the solution $U(r, x)$ of problem (2.1) with accuracy guaranteed by the inequality

$$
\left\|U(r, x)-S_{s, 2^{J}}\left(r, x ; U, \Psi^{-1}\right)\right\|_{C\left(K_{1}\right)} \leq \frac{\pi}{\sqrt{3}}\left(1+\left\|S_{s, 2^{J}}\right\|\right) E_{N_{\varepsilon, J}^{-}}\left(g_{1}\right)_{C_{2 \pi}},
$$

Estimates for norm of the operator $S_{s, 2^{J}}$ (interpolation projection onto the subspace $V_{j}^{s}$ ) can be found in Theorem from [2].

P r o o f. For convenience introduce the following notation:

$$
c_{j, k}\left(g_{1}\right)=\left(g_{1}(\cdot)-S_{s, 2^{j}}\left(\cdot ; g_{1}\right)\right)\left(x_{j+1}^{2 k+1}\right), \quad j \in \mathbb{N} \cup\{0\}, \quad k=\overline{0,2^{j}-1} .
$$

Using Euler's formula, we can represent (2.6) in the form

$$
U(r, x)=U(0,0)+\sum_{j=0}^{+\infty} \sum_{k=0}^{2^{j}-1} c_{j, k}\left(g_{1}\right) 2^{-j} \sum_{\nu \in \Delta_{\varepsilon}^{j+1} \cap \mathbb{N}} \widehat{\varphi}_{s}\left(\frac{\nu}{2^{j+1}}\right) \frac{r^{\nu}}{\nu} \cos \left(\nu\left(x-\frac{2 \pi(2 k+1)}{2^{j+1}}\right)\right)
$$


and partial sum $S_{s, 2^{J}}\left(r, x ; U, \Psi^{-1}\right)$ in the form

$$
\begin{gathered}
S_{s, 2^{J}}\left(r, x ; U, \Psi^{-1}\right)=U(0,0)+\sum_{j=0}^{J-1} \sum_{k=0}^{2^{j}-1} c_{j, k}\left(g_{1}\right) 2^{-j} \times \\
\times \sum_{\nu \in \Delta_{\varepsilon}^{j+1} \cap \mathbb{N}} \widehat{\varphi}_{s}\left(\frac{\nu}{2^{j+1}}\right) \frac{r^{\nu}}{\nu} \cos \left(\nu\left(x-\frac{2 \pi(2 k+1)}{2^{j+1}}\right)\right) .
\end{gathered}
$$

Note that the following representations hold

$$
\begin{gathered}
U(r, x)=\frac{1}{\pi} \int_{0}^{2 \pi} \sum_{\mu=1}^{+\infty} \frac{\cos (\mu(x-\xi))}{\mu}\left(U(1,0)+\sum_{j=0}^{+\infty} \sum_{k=0}^{2^{j}-1} c_{j, k}\left(g_{1}\right) \Phi_{s}^{j+1,2 k+1}(r, \xi)\right) d \xi, \\
S_{s, 2^{J}}\left(r, x ; U, \Psi^{-1}\right)=\frac{1}{\pi} \int_{0}^{2 \pi} \sum_{\mu=1}^{+\infty} \frac{\cos (\mu(x-\xi))}{\mu}\left(U(1,0)+\sum_{j=0}^{J-1} \sum_{k=0}^{2^{j}-1} c_{j, k}\left(g_{1}\right) \Phi_{s}^{j+1,2 k+1}(r, \xi)\right) d \xi .
\end{gathered}
$$

It follows from

$$
\begin{gathered}
\frac{1}{\pi} \int_{0}^{2 \pi} \sum_{\mu=1}^{+\infty} \frac{\cos (\mu(x-\xi))}{\mu} \Phi_{s}^{j+1,2 k+1}(r, \xi) d \xi= \\
=\frac{1}{\pi} \int_{0}^{2 \pi} \sum_{\mu=1}^{N_{\varepsilon, j}^{+}} \frac{\cos (\mu(x-\xi))}{\mu} 2^{-j} \sum_{\nu \in \Delta_{\varepsilon}^{j+1} \cap \mathbb{Z}^{+}} \widehat{\varphi}_{s}\left(\frac{\nu}{2^{j+1}}\right) r^{\nu} \frac{\cos \left(\nu\left(\xi-\frac{2 \pi(2 k+1)}{2^{j+1}}\right)\right)}{\nu} d \xi= \\
=2^{-j} \sum_{\nu \in \Delta_{\varepsilon}^{j+1} \cap \mathbb{N}} \widehat{\varphi}_{s}\left(\frac{\nu}{2^{j+1}}\right) \frac{r^{\nu}}{\nu} \cos \left(\nu\left(x-\frac{2 \pi(2 k+1)}{2^{j+1}}\right)\right)=\Psi_{s}^{j, k,-1}(r, x),
\end{gathered}
$$

where $N_{\varepsilon, j}^{+}=\left\lceil 2^{j}(1+\varepsilon)\right\rceil$ and the second equality holds in view of

$$
\begin{aligned}
& \frac{1}{\pi} \int_{0}^{2 \pi} \cos (\mu(x-\xi)) \cos \left(\nu\left(\xi-\frac{2 \pi(2 k+1)}{2^{j+1}}\right)\right) d \xi= \\
= & \delta_{\mu, \nu} \cos \left(\nu\left(x-\frac{2 \pi(2 k+1)}{2^{j+1}}\right)\right), \nu, \mu \in \Delta_{\varepsilon}^{j+1} \cap \mathbb{Z}^{+} .
\end{aligned}
$$

Let $S_{s, 2^{J}}\left(r, x ; g_{1}\right)$ be a partial sum of series in $(2.4)$, then

$$
\begin{gathered}
\left.\left|U(r, x)-S_{s, 2^{J}}\left(r, x ; U, \Psi^{-1}\right)\right|=\mid \frac{1}{\pi} \int_{0}^{2 \pi} \sum_{\mu=1}^{+\infty} \frac{\cos (\mu(x-\xi))}{\mu}\left(g_{1}(r, \xi)\right)-S_{s, 2^{J}}\left(r, \xi ; g_{1}\right)\right) d \xi \mid \leq \\
\left.\leq \frac{1}{\pi} \int_{0}^{2 \pi}\left|\sum_{\mu=1}^{+\infty} \frac{\cos (\mu(x-\xi))}{\mu}\right| \cdot \mid g_{1}(r, x)\right)-S_{s, 2^{J}}\left(r, x ; g_{1}\right) \mid d \xi \leq \\
\left.\leq \frac{1}{\pi}\left(\int_{0}^{2 \pi} 1 d \xi\right)^{1 / 2}\left(\int_{0}^{2 \pi}\left|\sum_{\mu=1}^{+\infty} \frac{\cos (\mu(x-\xi))}{\mu}\right|^{2} d \xi\right)^{1 / 2} \| g_{1}(x)\right)-S_{s, 2^{J}}\left(r, x ; g_{1}\right) \|_{C(2 \pi)}= \\
\left.=\frac{1}{\pi} \sqrt{2 \pi}\left(\pi \sum_{\mu=1}^{+\infty} \frac{1}{\mu^{2}}\right)^{1 / 2} \| g_{1}(x)\right)-S_{s, 2^{J}}\left(r, x ; g_{1}\right) \|_{C(2 \pi)} \leq \frac{\pi}{\sqrt{3}}\left(1+\left\|S_{s, 2^{J}}\right\|\right) E_{N_{\varepsilon, J}}\left(g_{1}\right)_{C_{2 \pi}},
\end{gathered}
$$


where the first equality follows from (2.7), the second equality follows from Parseval's identity, the second inequality follows from Hölder's inequality and the last inequality follows from Theorem in [2]. As the final result we have

$$
\left\|U(r, x)-S_{s, 2^{J}}\left(r, x ; U, \Psi^{-1}\right)\right\|_{C\left(K_{1}\right)} \leq \frac{\pi}{\sqrt{3}}\left(1+\left\|S_{s, 2^{J}}\right\|\right) E_{N_{\varepsilon, J}^{-}}\left(g_{1}\right)_{C_{2 \pi}} .
$$

\section{Conclusion}

Theorem 1 gives the solution (2.6) (up to an additive constant) of the problem (2.1) in form of uniformly convergent in $\bar{K}_{1}$ series of harmonic interpolating $2 \pi$-periodic wavelets. In this case, coefficients of series in (2.6) have an easy-to-calculate form in preference to calculating coefficients (integrals) in case of implementing orthogonal $2 \pi$-periodic wavelets. This useful fact simplify the numerical implementation of the suggested method.

\section{REFERENCES}

1. Subbotin Yu.N., Chernykh N.I. Harmonic wavelets in boundary value problems for harmonic and biharmonic functions. Proc. Steklov Inst. Math., 2011. Vol. 273, Suppl. 1. P. 142-159. DOI: $10.1134 /$ S0081543811050154

2. Subbotin Yu. N., Chernykh N. I. Interpolation Wavelets in Boundary Value Problems. Proc. Steklov Inst. Math., 2018. Vol. 300, Suppl. 1. P. 172-183. DOI: 10.1134/S0081543818020177

3. Subbotin Yu. N., Chernykh N.I. Interpolating-orthogonal wavelet systems. Proc. Steklov Inst. Math., 2009. Vol. 264, Suppl. 1. P. 107-115. DOI: 10.1134/S0081543809050083

4. Meyer Y. Ondelettes et opérateurs. Vol. I-III. Paris: Herman, 1990.

5. Offin D., Oskolkov K. A note on orthonormal polynomial bases and wavelets. Constr. Approx. 1993. Vol. 9. P. 319-325. DOI: $10.1007 /$ BF01198009

6. Tikhonov A. N., Samarskii A. A. Equations of Mathematical Physics. New York: Dover Publications, 1990. 